\section{SAT0753-HPR AWARENESS OF POSSIBLE SIDE EFFECTS OF NSAIDS AMONG THE ALBANIAN PATIENT POPULATION}

E. Pistja, A. Themeli. Continuing Education, Medical Training Center Santa Maria, Lezhe, Albania

Background: No official published figures are available regarding the annual use of NSAIDs in Albania. Nonsteroidal anti-inflammatory drugs are used primarily to manage different pain conditions, less commonly they are used for their antipyretic effect. Although generally well tolerated, conventional NSAIDs have been associated with a wide range of adverse effects. The most common of which are gastrointestinal tract (GIT) side effects like: dyspepsia, abdominal pain, heartburn, and the most serious life-threatening gastrointestinal (GI) ulceration. Objectives: To investigate patient awareness of the proper use and frequency of side effects in nonsteroidal anti-inflammatory drugs (NSAIDs) patients in Albania. Methods: This study was a prospective 15 question interview of patients purchasing medications, during randomized 1 hour/day pharmacy visits over a one month study period (May 2015).

The study was conducted in 4 community pharmacies located in the city of Tirana (capital of Albania). Two hundred and ten patients were included in this study. Results: Overall NSAIDs use during last year was $63 \%$ : Ibuprofene and diclofenac was the most used NSAIDs.

The majority of patients (58\%) reported having side effects upon NSAIDs-use; gastrointestinal upset was the most frequently reported side effect.

Patients' awareness regarding proper NSAIDs use was poor, and pharmacist role in counseling was inadequate.

However, user ability to discover the most common side effect to the drug seemed not to be affected.

Conclusions: Nonsteroidal anti-inflammatory drugs use awareness and knowledge of probable serious side effects and how to handle them was not adequate. This probably reflected on high incidence of side effects. Nonsteroidal antiinflammatory drugs are available on prescription as well as over the counter drugs.

Pharmacist involvement in education of patients using them is highly recommended and much needed to help decrease frequency of side effects.

However this is a small scale study and further studies need to be done.

References:

[1] Langman MJ. Risks of anti-inflammatory drug-associated damage. Inflamm Res 1999; 48: 236-238.

[2] Garcia Rodriquez LA, Jick H. Risk of upper gastrointestinal bleeding and perforation associated with individual non-steroidal anti-inflammatory drugs. Lancet 1994; 343: 769-772.

[3] Kay EA, Barker AR. Rheumatoid arthritis andosteoarthritis. In: Walker R, Edward C, editor. Clinicalpharmacy and therapeutics. Edinburgh (UK): Churchill Livingstone; 2002. p. 779-795.

Disclosure of Interest: None declared

DOI: 10.1136/annrheumdis-2017-eular.1089

\section{SAT0754-HPR "A NECESSARY INVESTMENT IN FUTURE HEALTH". PERCEPTIONS OF PHYSICAL ACTIVITY MAINTENANCE AMONG PEOPLE WITH RA PARTICIPATING IN AN OUTSOURCED HEALTH-ENHANCING PHYSICAL ACTIVITY PROGRAM}

E. Swärdh ${ }^{1,2}$, B. Nordgren ${ }^{1,2}$, C.H. Opava ${ }^{1,3}$, I. Demmelmaier ${ }^{1} .{ }^{1}$ Department of Neurobiology, Care sciences and Society, Division of Physiotherapy, Karolinska Institutet; ${ }^{2}$ Functional Area Occupational Therapy \& Physiotherapy, Allied Health Professionals' Function, Karolinska University Hospital, Huddinge; ${ }^{3}$ Department of Rheumatology, Karolinska University Hospital, Solna, Sweden

Background: Health-enhancing physical activity (HEPA) is an active treatment in rheumatoid arthritis (RA) that may be difficult for patients to maintain over time. A two-year HEPA program including twice-weekly circuit training at public gyms and 150 weekly minutes of moderately intense aerobic exercise was conducted in Sweden between 2011 and 2013. Regular peer support sessions were held to guide adoption and maintenance of HEPA. Patient perceptions on maintained physical activity in RA have been minimally explored.

Objectives: To describe perceptions of physical activity maintenance during the second year of an outsourced 2-year HEPA-program among people with RA Methods: A descriptive design with a qualitative inductive approach was used. Interviews were conducted with 18 participants with RA, including men and women differing in age, disease duration, activity limitation, perceived pain, levels of physical activity, training centers and peer support groups. Qualitative content analysis was used, and a pattern of theme, subthemes, categories, and subcategories was constructed based on the participants' perceptions of the phenomenon.

Results: A main overarching theme and three subthemes were established, called ' $A$ necessary investment in future health through dedication, affinity and awareness', which described participants' experiences of maintenance during the second year of an outsourced 2-year HEPA-program. This was further described in eight categories with 16 subcategories. The categories described the participants'; 'mindsets, habits, commitments, social support, PA contexts, monitoring, insights in PA, and health gains'.

Conclusions: The findings are partly in line with the theoretically derived explanations for maintenance of behavior change formulated to date, such as maintenance motives (self-determination and identity), self-regulation (skills and processes, lapse, relapse and coping) habits, resources (psychological and physical) as well as environmental and social influences. The results of this study could also be transferable to similar groups of people with RA in similar settings and useful in designing future HEPA interventions to facilitate maintenance of behavior change.

Disclosure of Interest: None declared

DOI: 10.1136/annrheumdis-2017-eular.6219

\section{SAT0755-HPR VARIATION IN SLE-RELATED PAIN: A SEVEN YEAR FOLLOW-UP STUDY}

E. Waldheim ${ }^{1}$, S. Ajeganova ${ }^{1}$, S. Bergman ${ }^{2}$, J. Frostegård ${ }^{1}$, E. Welin Henriksson ${ }^{3}$. ${ }^{1}$ Karolinska Institutet, Stockholm; ${ }^{2}$ University of Gothenburg, Gothenburg; ${ }^{3}$ Linköping Universitet, Linköping, Sweden

Background: In a previous study we have shown that $24 \%$ of patients with SLE in our cohort reported high level of SLE-related pain, $\geq 40 \mathrm{~mm}$ on VAS $(0-100$ $\mathrm{mm}){ }^{1}$ These patients with high pain level also reported significantly more fatigue, anxiety and depression and reduced health-related quality of life compared to the SLE patients with low pain level, $\leq 39 \mathrm{~mm}$ on VAS. ${ }^{2}$

Objectives: To investigate the variation in self-reported SLE-related pain and its association with presence of chronic widespread pain (CWP) and patient-related outcomes after seven years of follow-up.

Methods: 64 of 84 patients agreed to participate in the 7-year follow-up and answered questionnaires on pain (VAS/mm), fatigue (MAF), HRQoL (SF-36), anxiety and depression (HADS) and, in case of remaining pain > three months, marked painful body regions on a pain-drawing. Disease activity and damage (SLAM, SLEDAI, SLICC) were also captured. Nonparametric statistics were used to compare the different groups. Difference in measures (diff) between inclusion and follow-up was calculated.

Results: For the patients with low degree of SLE-related pain the previous week ( $\leq 39 \mathrm{~mm}$ on VAS) at inclusion, $n=50$, there were no significant difference at 7 years follow-up in pain, fatigue, anxiety, depression and all dimensions of SF-36, except for deterioration in physical function median diff (IQR) 0 (-10 to 5), $p=0.024$. Of these patients with low level of pain, $26 \%$ indicated chronic widespread pain on the pain drawing.

Among patients with high degree of pain ( $\geq 40 \mathrm{~mm}$ on VAS) at inclusion, $n=14$, half of the patients reported significantly decreased pain, diff (IQR) 45 (35 to 65), $\mathrm{p}=0.021$, fatigue, 8 ( 8 to 17 ), $\mathrm{p}=0.018$, anxiety, 4 ( 1 to 4 ), $\mathrm{p}=0.035$ and depression, 4 ( 2 to 5 ), $p=0.018$ and improvements in all dimensions of SF-36 except for role emotional and social function at follow-up, $p<0.05$.

However, half of the patients with high degree of pain at inclusion reported no significant changes at follow up regarding pain, median diff (IQR) -13 (-20 to 28), fatigue, 5 ( -0.3 to 6$)$, anxiety, 2 ( -1 to 3 ) and depression, 0 ( -3 to 2$)$. These patients reported significantly deterioration in vitality in SF-36, diff (IQR) 20 (15 to 35), $\mathrm{p}=0.0018$ but no significant changes in the other dimensions of SF-36. All patients with high levels of remaining pain indicated chronic widespread pain on the pain drawing. These patients with remaining pain had significantly higher SLAM at follow-up compared to the patients with decreased pain at follow-up, $p=0.017$ and the patients with low levels of pain at inclusion, $p=0.006$. No significant differences were found regarding SLEDAI and disease damage.

Conclusions: Self-reported level of disease-related pain remain low in most patients and in some patients also significantly reduced. However, half of the patients with high level of pain at inclusion still experienced high level of pain and pain-related problems including widespread pain after 7 years of follow-up. These results suggest a transition from SLE-related pain to chronic widespread pain, which requires different pain management.

References:

[1] Waldheim,E.,et al.(2013).Lupus 22:136-143.

[2] Waldheim,E.,et al.(2013).Lupus 22:1118-1127.

Disclosure of Interest: None declared

DOI: 10.1136/annrheumdis-2017-eular.1446

\section{SAT0756-HPR EFFECT OF NSAID INTAKE ON KINESIOPHOBIA IN PATIENTS WITH ANKYLOSING SPONDYLITIS}

G.I. Kinikli ${ }^{1}$, S. Karahan ${ }^{2}$, A. Ates ${ }^{3}$, M. Turgay ${ }^{3}$, G. Kinikli ${ }^{3} .{ }^{1}$ Department of Physiotherapy and Rehabilitation, Hacettepe University Faculty of Health Sciences; ${ }^{2}$ Department of Biostatistics, Hacettepe University Faculty of Medicine; ${ }^{3}$ Department of Rheumatology, Ankara University Faculty of Medicine, Ankara, Turkey

Background: Spinal stiffness and loss of spinal mobility, explained byspinal inflammation and structural damage due to extensive osteoproliferation, are characteristics of Ankylosing Spondylitis (AS). AS usually disables a person with severe back pain and, in later stages, remarkable spinal kyphotic deformity. The deformity eventually may necessitate a major corrective procedure. Therefore, controlling the symptoms and progression of AS in early stages by effective medication is the main step in the management of AS.

Objectives: The aim of this study was to investigate the effectiveness of DMARD therapies on NSAID intake and kinesiophobia in patients with AS. 